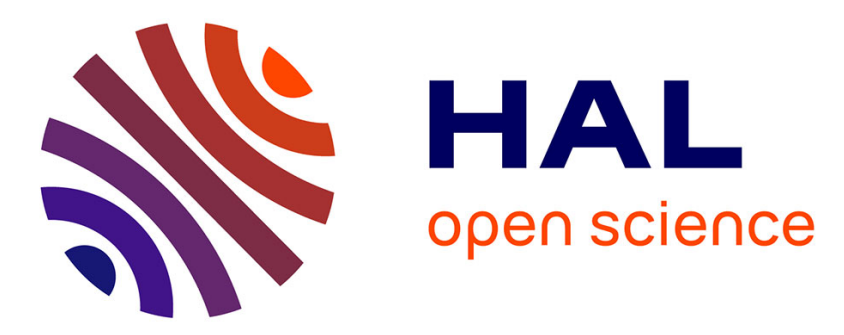

\title{
THEORIES OF STATIC AND DYNAMIC CRITICAL PHENOMENA AT ORDER-DISORDER AND UNMIXING TRANSITIONS
}

\author{
K. Binder
}

\section{- To cite this version:}

K. Binder. THEORIES OF STATIC AND DYNAMIC CRITICAL PHENOMENA AT ORDERDISORDER AND UNMIXING TRANSITIONS. Journal de Physique Colloques, 1977, 38 (C7), pp.C7396-C7-403. 10.1051/jphyscol:1977780 . jpa-00217283

HAL Id: jpa-00217283

https://hal.science/jpa-00217283

Submitted on 1 Jan 1977

HAL is a multi-disciplinary open access archive for the deposit and dissemination of scientific research documents, whether they are published or not. The documents may come from teaching and research institutions in France or abroad, or from public or private research centers.
L'archive ouverte pluridisciplinaire HAL, est destinée au dépôt et à la diffusion de documents scientifiques de niveau recherche, publiés ou non, émanant des établissements d'enseignement et de recherche français ou étrangers, des laboratoires publics ou privés. 


\title{
THEORIES OF STATIC AND DYNAMIC CRITICAL PHENOMENA AT ORDER-DISORDER AND UNMIXING TRANSITIONS
}

\author{
K. BINDER \\ Theoretische Physik, Universitat, 66 Saarbrücken 11, W-Germany.
}

\begin{abstract}
Résumé. - Une brève revue de quelques concepts récents sur les transformations de phase dans les solides est donnée. Tout d'abord, des modèles de réseaux microscopiques simples sont introduits, des modèles d'Ising qui décrivent soit la démixtion (si le paramètre d'ordre de la transition est conservé) soit l'ordre (si le paramètre d'ordre n'est pas conservé). On montre que les approximations de Bragg-Williams ou de champ moyen décrivent de façon imprécise la transition parce qu'elles négligent les fluctuations, sauf dans le cas des forces à longue distance (hydrogène dans les métaux etc.). Tandis que les calculs de simulation Monte Carlo donnent une description assez précise dans tout le domaine de température, des informations très précises sur les exposants critiques sont obtenues par la méthode groupe de renormalisation basé sur la notion d'échelle. Par l'approche de cluster dynamics ces idées sont étendues aux phénomènes cinétiques loin de l'équilibre.
\end{abstract}

\begin{abstract}
Some recent concepts on phase transformations in solids are briefly reviewed. First simple microscopic lattice models are introduced, kinetic Ising models which describe either unmixing (if the order parameter of the transition is conserved) or ordering (if the order parameter is not conserved). Bragg-Williams or related meanfield-approximations are shown to describe the transition inaccurately due to the neglect of fluctuations, except for the case of long-range forces (hydrogen in metals etc.). While Monte Carlo computer simulations give a fairly accurate description over the whole temperature range, most accurate information on critical exponents is due to the renormalization group approach based on scafing ideas. By the cluster dynamics approach these ideas are extended also to kinetic phenomena far from equilibrium.
\end{abstract}

1. Introduction : phenomenology of phase transitions in alloys. - Recently much effort has been devoted to understand theoretically order-disorder phenomena and other phase transitions in solids : ferroelectrics [1] as well as other displacive structural phase transitions [2], magnets [3], molecular crystals like $\mathrm{NH}_{4} \mathrm{Cl}$ [4], $\mathrm{KCN}$ [5], where $\mathrm{NH}_{4}^{+}$-tetraedra or $\mathrm{CN}^{-}$-dumbells order in preferred directions, interstitial alloys like hydrogen in metals $[6,7]$ etc. In this review, however, we consider only «substitutional » binary alloys like $\mathrm{CuZn}, \mathrm{ZnAl}$, etc. [8]. In such AB-systems one often finds one of the following two behaviors (figure 1) : (i) a miscibility gap occurs (for temperatures $T$ below $T_{\mathrm{c}}$ there is a range of concentrations $c_{\mathrm{B}}$, in which the equilibrium consists of two coexisting phases). (ii) Below $T_{\mathrm{c}}$ a superstructure appears (A atoms preferently occupy sublattice I, B-atoms sublattice II). In the case of unmixing (figure $1 \mathrm{~A})$ a state $\left(c_{\mathrm{B}}, T\right)$ below the coexistence curve $c_{\mathrm{B}}^{(1),(2)}(T)$ is described by a coexistence of two phases with concentrations $c_{B}^{(1)}(T)$ and $c_{B}^{(2)}(T)$, their respective amounts being given by the lever rule. Lowering $T$ and crossing the coexistence curve at fixed $c_{\mathrm{B}}$, the transition is always of first order except for the critical concentration $c_{\mathrm{B}}^{\text {crit }}$, for which it is of second order. The order parametes $\langle\Psi\rangle$ of this transitions is $\langle\Psi\rangle=c_{\mathrm{B}}^{(2)}(T)-c_{\mathrm{B}}^{(1)}(T)$.
In the case of ordering the transition may be of second (figure 1B, e.g. CuZn) or first order (figure 1C, e.g. $\mathrm{AuCu}$ ). In the 2 nd order case the order parameter $\langle\Psi\rangle=c_{\mathrm{B}}^{\mathrm{II}}(T)-c_{\mathrm{B}}^{\mathrm{S}}(T)$ continuously increases as $T$ is lowered beyond $T_{\mathrm{c}}\left(c_{\mathrm{B}}\right)$, and only one-phase states occur. For the 1 st order transition two cases exist : 1) At $c_{B}^{\text {crit }}$ where the transition temperature has a maximum (or minimum) the order parameters jumps discontinuously from zero in the disordered phase to a nonzero value in the ordered phase. 2) For $c_{B}=c_{B}^{\text {crit }}$ the critical line splits into two branches $T_{\mathrm{c}}^{(1)}\left(c_{\mathrm{B}}\right)$, $T_{\mathrm{c}}^{(2)}\left(c_{\mathrm{B}}\right)$, between which the ordered and disordered phases coexist. Since their amounts are again given by the lever rule, the order parameter varies linearly with temperature there.

The phase diagrams of real materials may be much more complicated, since several of the cases figure 1A-C may occur for one system $\mathrm{AB}$. Combination of these phase diagram elements leads to new features like tricritical points and triple points (figure 2). At a tricritical point $\left(c_{\mathrm{B}}^{\mathrm{t}}, T_{\mathrm{t}}\right)$ the order of the transition changes from second to first order [9]. At a triple point three phases coexist, for instance one disordered and two different ordered ones (for instance, CuAu and $\mathrm{Cu}_{3} \mathrm{Au}$ in the $\mathrm{Cu}-\mathrm{Au}$ system).

We now will discuss (i) simple lattice models of alloys and the relation of the phase diagram to basic 

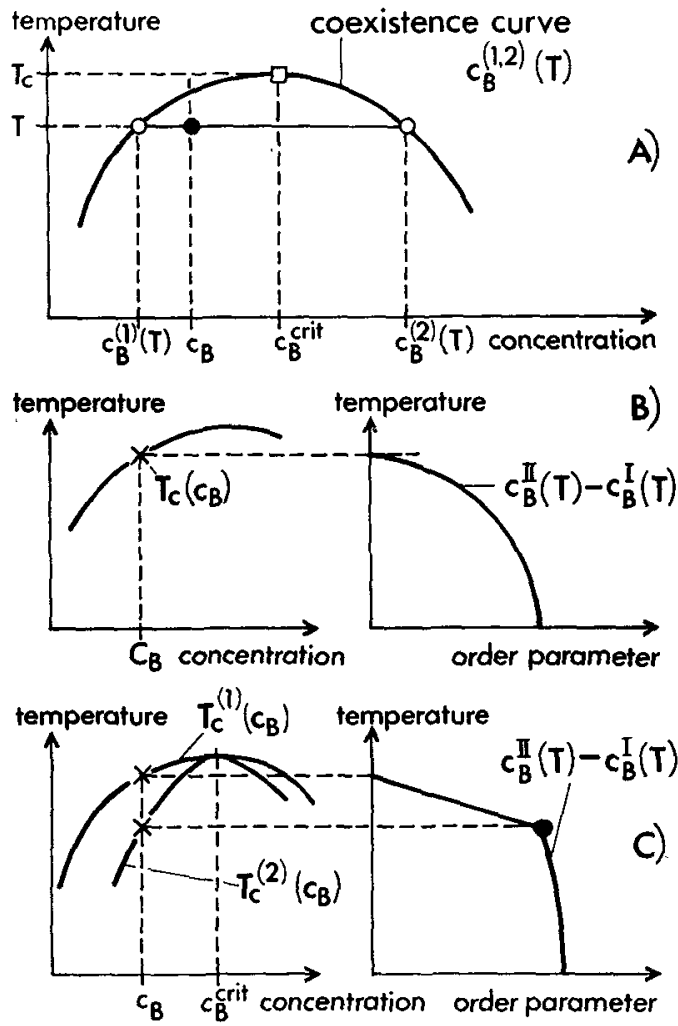

FIG. 1A. - Schematic phase diagram of an alloy with a miscibility gap; B, C) Phase diagram and temperature dependence of the order parameter of superstructure alloys with second (B) and first (C) order transition.
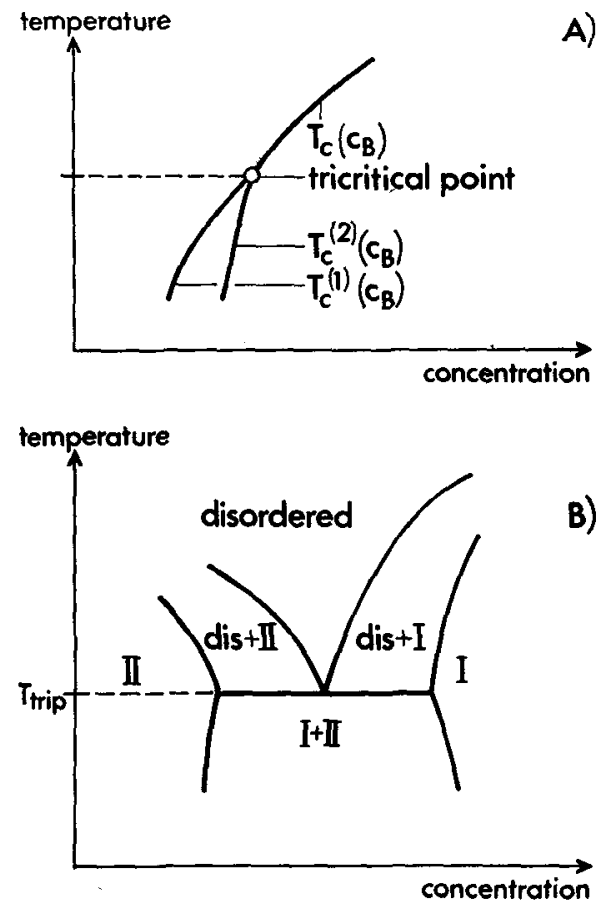

FIG. 2 A. - Schematic phase diagram of an ordering alloy close to a tricritical point; B) Phase diagram close to a triple point. interaction parameters (ii) predictions for static and dynamic critical properties (iii) kinetics of the first order transitions.

2. Generalized Ising models and the calculation of phase diagrams. - (i) We describe the distribution of two species $\mathrm{A}, \mathrm{B}$ on the sites $\{i\}$ of a perfect crystal lattice by local concentration variables $\left\{c_{i}\right\}\left(c_{i}=1\right.$ if $i$ is occupied by $\mathrm{B}, c_{i}=0$ otherwise). Then the effective Hamiltonian, which depends on both the $\left\{c_{i}\right\}$ and the atomic momenta $\left\{\mathbf{p}_{i}\right\}$ and displacements $\left\{\mathbf{u}_{i}\right\}$, is written as

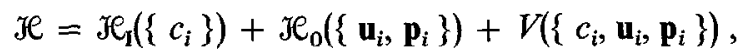

where $V$ denotes the coupling between the various degrees of freedom. Assuming $\mathfrak{H}_{\Gamma}$ " $V$ one may calculate the partition function neglecting $V$; i.e., averages $\left\langle\mathbf{A}\left(\left\{c_{i}\right\}\right)\right\rangle$ are calculated using the generalized Ising Hamiltonian $\mathfrak{H}_{\mathrm{I}}\left(\left\{c_{i}\right\}\right)$ alone. The only effect of the coupling $V$ between the configurational degrees of freedom and the kinetic energy of the lattice (contained in $\mathfrak{H}_{0}$ ) will be to introduce dynamics in the system by random interchanges $c_{i} \rightleftharpoons c_{j}$ between neighboring sites. We will disregard the fact that this interchange occurs indirectly via vacancies rather than directly and also neglect the possible influence of defects on bulk static properties.

Due to the lattice misfit between the atomic radii of $A$ and $B$ and the resulting elastic distortions $V$ often will not be negligibly small. Treating $V$ in a perturbation expansion and performing the trace over the variables $\left\{\mathbf{u}_{i}, \mathbf{p}_{i}\right\}$, one is left with a renormalized $\mathfrak{H}_{\mathrm{I}}$ which now describes a compressible generalized Ising model [7]. Such models successfully apply to $\mathrm{H}$ in metals [7] where the elastic interaction completely dominates. But for systems where short range forces are important only rather unrealistic special cases have been solved [10]. Thus we neglect the elastic interaction in all what follows.

Next we expand $\mathfrak{H}_{1}$ in the $c_{i}$ 's, omitting a constant and using translational invariance

$$
\begin{aligned}
\mathscr{H}_{\mathrm{I}}=\left(\mu_{\mathrm{A}}-\right. & \left.\mu_{\mathrm{B}}\right) \sum_{i} c_{i}+\sum_{i \neq j}\left[c_{i} c_{j} \psi_{\mathrm{BB}}\left(\mathbf{r}_{i j}\right)+\right. \\
& +2 c_{i}\left(1-c_{j}\right) \psi_{\mathrm{AB}}\left(\mathbf{r}_{i j}\right) \\
& \left.+\left(1-c_{i}\right)\left(1-c_{j}\right) \psi_{\mathrm{AA}}\left(\mathbf{r}_{i j}\right)\right]+\ldots .
\end{aligned}
$$

Here $\mu_{\mathrm{A}}, \mu_{\mathrm{B}}$ are chemical potentials, $\psi_{\mathbf{B B}}\left(\mathbf{r}_{i j}\right)$ is the effective pair potential between two $\mathrm{B}$-atoms at sites $i, j$, etc. Although $c_{i}(=0,1)$ is not a small parameter, terms of order $c_{i} c_{j} c_{k}$ etc. are usually neglected in Eq. (1), which then is precisely equivalent to an Ising magnet : With pseudospins $\sigma_{i}= \pm 1$ putting $c_{i}=\left(1-\sigma_{i}\right) / 2 \mathrm{Eq}$. (1) becomes (omitting a constant)

$$
\begin{aligned}
\mathscr{H}_{\mathrm{I}}=-\sum_{i \neq j} J\left(\mathbf{r}_{i j}\right) \sigma_{i} \sigma_{j}- & H \sum_{i} \sigma_{i}= \\
& =-\sum_{i} H^{\mathrm{eff}}\left(\left\{\sigma_{j}\right\}\right) \sigma_{i},
\end{aligned}
$$


with an exchange $J=\psi_{\mathrm{AB}} / 2-\left(\psi_{\mathrm{AA}}+\psi_{\mathrm{BB}}\right) / 4$ and a magnetic field $H=\left[\mu_{\mathrm{A}}-\mu_{\mathrm{B}}+\sum_{j}\left(\psi_{\mathrm{BB}}-\psi_{\mathrm{AA}}\right)\right] / 2$. Since Eq. (2) is invariant under $\sigma_{i} \rightarrow-\sigma_{i} . H \rightarrow-H$, the phase diagram in the $(T, H)$ plane is symmetric around $\langle\sigma\rangle=0$. The corresponding alloy diagram is symmetric around $c_{B}=1 / 2$. One often finds [11] quite asymmetric diagrams, indicating that the above neglect of higher order terms in Eq. (1) is not justified. Such terms have been included [12] so far by variants of the mean-field theory (Kikuchi approximations [13]) only, which predict critical properties unreliably due to the neglect of statistical fluctuations. Thus we disregard these higher order terms here.

For the computation of the phase diagram from Eq. (2), three approximate methods exist : (i) meanfield (or Bragg-Williams) approximations and variants thereof [13-15]. In Eq. (2) one replaces the (fluctuating) field $H^{\text {eff }}\left(\left\{\sigma_{j}\right\}\right)$ by its average

$$
H_{\alpha}^{\text {eff }}=H+\sum_{j} J_{i j}\left\langle\sigma_{j}\right\rangle
$$

on each sublattice $\alpha$ and calculates the $\left\langle\sigma_{j}\right\rangle$ for all sublattices selfconsistently. Figure 3 shows an example [16] for a square lattice with repulsive interaction both between nearest $\left(J_{\mathrm{NN}}<0\right)$ and next nearest $\left(J_{\mathrm{NNN}}<0\right)$ neighbors. Such a system may model order-disorder transitions in adsorbed monolayers at surfaces [17].

(ii) Monte Carlo computer simulations [18]. There one constructs a random walk through the phase space of the $N$-particle model system. Averaging over this Markov chain yields the exact statistical mechanics of the system, apart from statistical errors due to finite-time averaging. In principle, these errors can be made arbitrarily small if only enough computer time is invested. In practice sufficient accuracy is obtained for systems with fairly short-ranged interaction only. Even then one has to take care of finite size

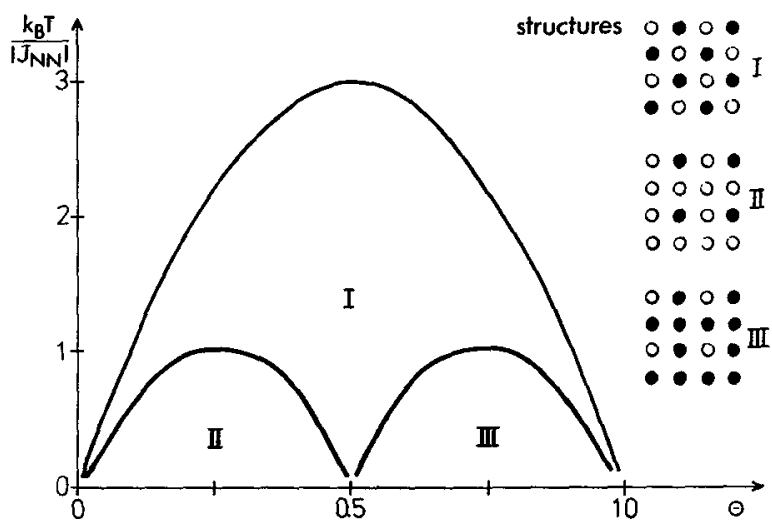

FIG. 3. - Temperature-concentration diagram for a model with three ordered phases, according to mean field theory. After Ref. [16]. and boundary effects [18] (in practice the method is limited to $N \approx 10^{3}-10^{5}$ ). While the accuracy thus can hardly compete with series extrapolations [19] or the renormalization group $[3,20]$, as far as critical exponents [19] are concerned, one obtains a fairly accurate description of the phase diagram. As example figure 4 gives the phase diagram for the same model as figure 3 . Note that mean field theory predicts all transitions to be of 2 nd rather than 1st order and thus misses the existence of the two tricritical and triple points, as well as the two-phase regions. It also greatly over-estimates the tendency to order. Even in more favorable cases where mean field theory gives the correct qualitative structure of the phase diagram, quantitative predictions of transition temperatures $k_{\mathrm{B}} T_{\mathrm{C}}\left(c_{\mathrm{B}}\right) /\left|J_{\mathrm{NN}}\right|$ etc. will still be inaccurate.

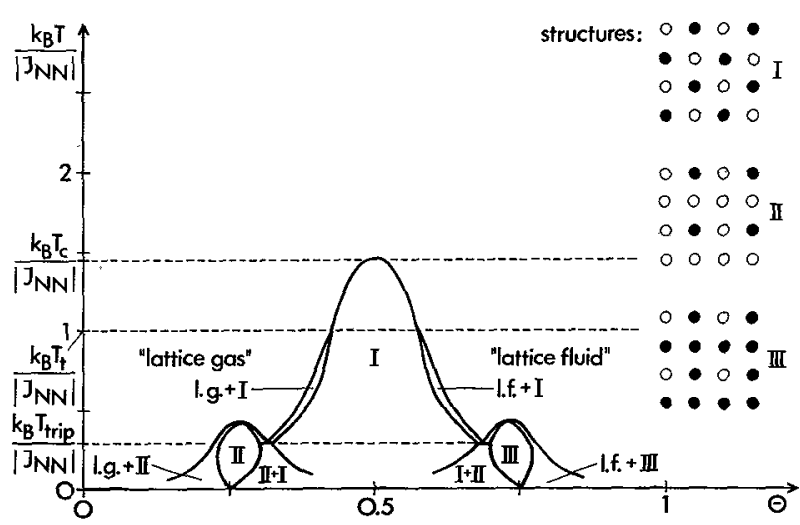

FIG. 4. - Same model as figure 3 but phase diagram obtained from Monte Carlo. After Ref. [16].

(iii) REAL-SPACE-RENORMALIZATION GROUP TRANSFORMATIONS [21-23]. - This method rests on the idea of scaling $[19,24]$. (figure 5). Since the order parameter correlation $G\left(\mathbf{r}_{i j}\right)=\left\langle\psi_{i} \psi_{j}\right\rangle$ becomes longranged close to the second order transition at $T_{\mathrm{c}}\left(c_{\mathrm{B}}\right)$, the correlation length $\xi$ is much larger than the lattice spacing and details on that scale do not matter. $G$ becomes a function of $\left|\mathbf{r}_{i j}\right| / \xi$ only, apart from a scale factor :

$$
G\left(\mathbf{r}_{i j}\right)=\left|\mathbf{r}_{i j}\right|^{-(d-2+\eta)} \tilde{G}\left(\left|\mathbf{r}_{i j}\right| / \xi\right), \quad\left|\mathbf{r}_{i j}\right|, \xi \rightarrow \infty .
$$

Here $d$ is the dimensionality of the system, $\eta$ a critical exponent and $\tilde{G}(x)$ a scaling function. Since details on the lattice scale don't matter we consider the problem of interacting cell spins $\left\{\sigma^{\prime}\right\}$ rather than the original one (figure 5). These problems would be equivalent if

$$
\begin{aligned}
& \exp \left[-\mathcal{H}_{\{J\}}\left(\left\{\sigma_{i}^{\prime}\right\}\right) / k_{\mathrm{B}} T\right] \equiv \\
& \equiv \sum_{\left\{\sigma_{i}\right\}} P\left(\left\{\sigma_{i}^{\prime}\right\},\left\{\sigma_{i}\right\}\right) \exp \left[-\mathscr{H}_{\{J}\left(\left\{\sigma_{i}\right\} / k_{\mathrm{B}} T\right)\right],
\end{aligned}
$$


where the projection $P$ makes the step $\left\{\sigma_{i}\right\} \rightarrow\left\{\sigma_{l}^{\prime}\right\}$ precise [e.g. majority rule [21] $\left.\sigma_{i}^{\prime}=\operatorname{sign} \sum_{i \in \mathrm{cell}} \sigma_{i}\right]$. Eq. (4) yields a renormalized effective Hamiltonian $\mathscr{H}_{\left\{J^{\prime}\right\}}\left(\left\{\sigma_{i}\right\}\right)$, the new couplings $\left\{J^{\prime}\right\}$ being expressed in terms of the old ones. Repeating this transformation $\mathfrak{H}_{\left\{J_{\}}\right\}}=\hat{R} \mathcal{H}_{\left\{\eta_{\}}\right.}$many times the problem is simplified eliminating degrees of freedom step by step. Treating Eq. (4) rigorously the new Hamiltonian would each step become more complicated, of course. But the idea now is that close to $T_{c}$ details of the Hamiltonian do not matter for the critical behavior (universality [25]) : hence we approximate (Eq. (4)) using only those parts of $\mathscr{H}_{\left\{J^{\prime}\right\}}$ which have the same structure as $\mathfrak{H}_{\left\{\eta_{\}}\right.}$(e.g., always only pairwise interactions). Then Eq. (4) easily is iterated :

$$
\{J\} \rightarrow\left\{J^{\prime}\right\} \rightarrow\left\{J^{\prime \prime}\right\} \rightarrow \cdots \rightarrow\left\{\begin{array}{cc}
\left\{J^{*}\right\} & T=T_{\mathrm{c}} \\
0 & T>T_{\mathrm{c}}
\end{array}\right\}
$$

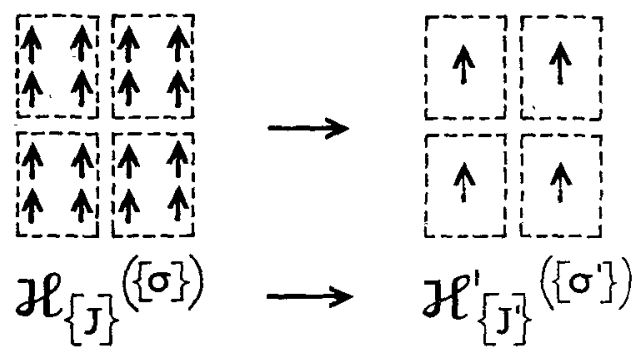

FIG. 5. - The Kadanoff method of introducing cell spins $\sigma^{\prime}$.

Right at $T_{\mathrm{c}}$, the renormalization leads to a nontrivial fixed point with couplings $\left\{J^{*}\right\}: \hat{R} \mathcal{H}_{\left\{J^{*}\right\}} \equiv \mathscr{H}_{\left\{J^{*}\right\}}$. It turns out that the eigenvalues of the renormalization group equation close to this fixed point are related to the critical exponents $[3,20,21]$. For $T>T_{\mathrm{c}}$, on the other hand, the cells ultimately become noninteracting, as their size exceeds $\xi$, and hence the desired partition function in Eq. (4) is calculated by a convergent iteration. Thus both estimates for critical exponents and the free energy away from $T_{\mathrm{c}}$ are obtained. So far most useful applications concern problems for $d=2$, like the triangular lattice with $J_{\mathrm{NN}}<0$ (including a three-spin-interaction) [23], and the square lattice [22] with $J_{\mathrm{NN}}<0, J_{\mathrm{NNN}} \neq 0$. While there the method is very accurate for $J_{\mathrm{NNN}}=0$, results [22], for $J_{\mathrm{NNN}} \neq 0$ are not so good : for $J_{\mathrm{NNN}}<0$, the phase diagram is qualitatively correct but inaccurate (e.g. for $J_{\mathrm{NNN}} / J_{\mathrm{NN}}=-1 / 2$ one has $[16,26] T_{\mathrm{c}} /\left|J_{\mathrm{NN}}\right| \approx 3.86$, $T_{\mathrm{t}} /\left|J_{\mathrm{NN}}\right| \approx 1.36$ rather than $[22] T_{\mathrm{c}} /\left|J_{\mathrm{NN}}\right| \approx 5$, $\left.T_{\mathrm{t}} /\left|J_{\mathrm{NN}}\right| \approx 1.1\right)$. For $J_{\mathrm{NNN}}<0$ the situation is even worse, since [27] the existence of structures (II, III) (figure 4) is not revealed. But it seems probable that more successful (and more sophisticated) variants of this method will be available soon.
3. Static critical and multicritical behavior. - Close to $T_{\mathrm{c}}\left(\right.$ or $T_{t}$ ) we expect singular behavior of $F$ and hence of the order parameter and its correlation :

$$
\begin{gathered}
F-F\left(T_{\mathrm{c}}\right) \propto\left|1-T / T_{\mathrm{c}}\right|^{2-\alpha}, \\
\langle\psi\rangle_{H_{\psi}=0} \propto\left(1-T / T_{\mathrm{c}}\right)^{\beta},\langle\psi\rangle_{T_{\circ}} \propto H_{\psi}^{1 / \delta}(6) \\
\chi_{\psi}=\frac{\partial\langle\psi\rangle}{\partial H_{\psi}} \propto\left|1-T / T_{\mathrm{e}}\right|^{-\gamma}, \quad \xi \propto\left|1-T / T_{\mathrm{c}}\right|^{-v},
\end{gathered}
$$

where $H_{\psi}$ is the field conjugate to the order parameter, and $\alpha, \beta, \gamma \ldots$ are critical exponents. At a tricritical point, $T_{\mathrm{c}}$ in Eq. (6) simply is replaced by $T_{t}$ and $\alpha, \beta, \gamma \ldots$ by $\alpha_{t}, \beta_{t}, \gamma_{t}, \ldots$ The simplest theory of critical behavior is the phenomenological Landau theory (for alloys its kinetic version is known as Cahn-Hilliard theory [28]). As in Eq. (4), short wavelength fluctuations are disregarded introducing a coarse-grained order parameter $\psi(\mathbf{r}, t) \equiv \sum_{i \in V} \sigma_{i}(t) / V \cdot \psi$ should be small for $T \rightarrow T_{\mathrm{c}}$ and hence the effective Hamiltonian $\mathscr{H}_{\mathrm{cg}}(\psi)$ is expanded in powers of $\psi$ and $\nabla \psi$, i.e. [for symmetry reasons no $\nabla \psi, \psi^{3}$ and $\psi^{5}$ terms occurs]

$$
\begin{aligned}
\mathscr{H}_{\mathrm{cg}}(\psi)=F_{\mathrm{cg}}(\psi)+\frac{1}{2} K(\nabla \psi)^{2} & = \\
=\frac{1}{2} A \psi^{2}+\frac{1}{4} B \psi^{4}+ & \frac{1}{6} C \psi^{6}-H_{\psi} \psi+ \\
& +\frac{1}{2} K(\nabla \psi)^{2}+F_{0},
\end{aligned}
$$

$F_{0}, A, B, C, K$ beeing constants. Landau's theory now assumes the free energy functional

$$
\exp \left[-F / k_{\mathrm{B}} T\right]=\operatorname{Tr}_{\{\psi(\mathbf{r})\}} \exp \left\{-\int \mathrm{dr} \mathscr{H}_{\mathrm{cg}}[\psi(\mathbf{r})] / k_{\mathrm{B}} T\right\}
$$

to be dominated by the term which minimizes the integral in Eq. (8), and neglects the other (fluctuation-) contributions to $F$. Thus we find $F=\mathfrak{H}_{\mathrm{cg}}(\psi)$, where $\psi$ is a solution of the Ginzburg-Landau equation $A \psi+B \psi^{3}+C \psi^{5}-K \nabla^{2} \psi=H_{\psi}$. At a critical point $H_{\psi}=0$ and $A\left(T_{\mathrm{c}}\right)=0$, hence $A(T)=a\left(T-T_{\mathrm{c}}\right)$, $B \neq 0$, and thus $\left(\nabla^{2} \psi=0\right.$ for homogeneous systems) $\psi=\left[a\left(T_{\mathrm{c}}-T\right) / B\right]^{1 / 2}, \beta=1 / 2$. Similarly one finds [19] $\alpha=0, \gamma=1, v=1 / 2$, and $\eta=0$. A tricritical point occurs if $B\left(T_{\mathrm{t}}\right)=A\left(T_{\mathrm{t}}\right)=0$; then $\psi=\left[a\left(T_{\mathrm{t}}-T\right) / C\right]^{1 / 4}$, $\beta_{t}=1 / 4$, and similarly $\alpha_{\mathrm{t}}=1 / 2, \gamma_{\mathrm{t}}=1$, and $v_{\mathrm{t}}=1 / 2$.

We now analyze the above neglect of fluctuations within the context of Landau theory itself (Ginzburg criterion [29]). First we obtain the response to a wavevector-dependent field $H_{\psi}(\boldsymbol{q})=H_{\psi} \exp [i q \mathrm{q}]$ above $T_{\mathrm{c}}$

$$
\begin{aligned}
\psi(\mathbf{q})=\chi(\mathbf{q}) H_{\psi}(\mathbf{q})= & {\left[A+K q^{2}\right]^{-1} H_{\psi}(\mathbf{q})=} \\
& =K^{-1}\left[\xi_{\mathrm{MF}}^{-2}+q^{2}\right]^{-1} H_{\psi}(\mathbf{q}) .
\end{aligned}
$$

Next we consider the Ginzburg-Landau equation for homogeneous fields above $T_{\mathrm{c}}$ \{ putting $C=0, \nabla^{2} \psi=0$, 
hence $\left.H_{\psi}=\psi\left[A+B \psi^{2}\right]\right\}$ and ask : do fluctuations give a weak, or strong, correction to the mean field susceptibility $A^{-1}$ ? We estimate $\left\langle\psi^{2}\right\rangle$ from the fluctuation relation [29]

$$
\begin{aligned}
&\left\langle\psi^{2}\right\rangle \propto \sum_{i j}\left\langle\psi_{i} \psi_{j}\right\rangle \propto \int \mathrm{d} \mathbf{q} \chi(\mathbf{q})= \\
&=K^{-1} \int \frac{\mathrm{d} \mathbf{q}}{q^{2}+\xi_{\mathrm{MF}}^{-2}}=K^{-1} \int \frac{\mathrm{d} \mathbf{q}}{q^{2}}- \\
&-K^{-1} \xi_{\mathrm{MF}}^{-2} \int \frac{\mathrm{d} \mathbf{q}}{q^{2}\left(q^{2}+\xi_{\mathrm{MF}}^{-2}\right)}
\end{aligned}
$$

The term $K^{-1} \int \mathrm{d} \mathbf{q} / q^{2}$ gives rise to a shift of $T_{\mathrm{c}}$. More interesting is the second term : if $d<4$, the main contribution to the integral comes from small $q$, one obtains a correction $\propto \xi_{\mathrm{MF}}^{2-d} \propto\left(T-T_{\mathrm{c}}\right)^{d / 2-1}$ : this correction becomes large in comparison with $A \propto\left(T-T_{\mathrm{c}}\right)$ as $T \rightarrow T_{\mathrm{c}}$ : the neglect of fluctuations and hence mean field theory break down. If $d>4$, the main contribution comes from large $q$, the correc-
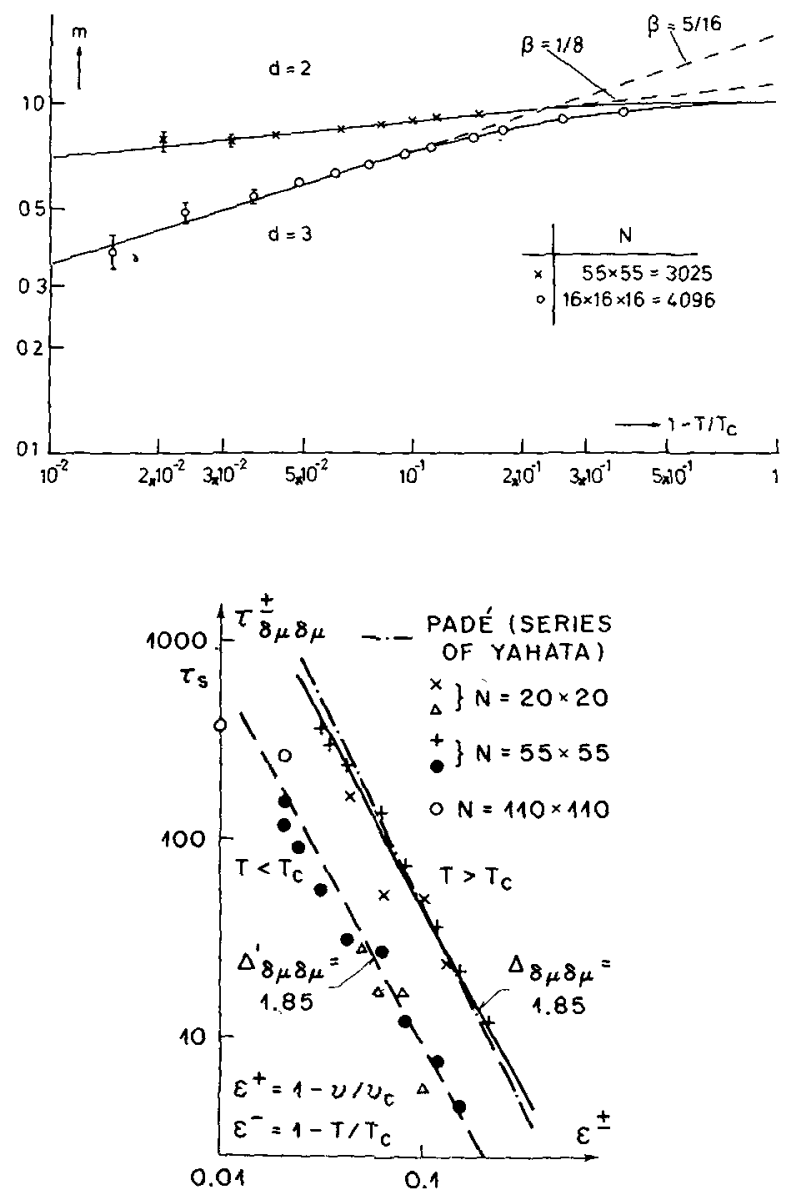

FIG. 6A. - Monte Carlo results for the order parameter of twoand threedimensional nearest neighbor Ising models. B) Monte Carlo results for the temperature dependence of the order parameter relaxation time. After Ref. [18]. tion is $\propto \xi_{\mathrm{MF}}^{-2} \propto\left(T-T_{\mathrm{c}}\right)$, i.e. of the same order as $A$ : thus only prefactors get renormalized, but the Landau critical exponents are o.k. At the marginal case $d^{*}=4$ one also gets Landau exponents but logarithmic correction factors [30]. At a tricritical point the fluctuations are due to the $C \psi^{5}$ rather the $B \psi^{3}$ term and then [31] $d^{*}=3$, again one gets Landau exponents apart from logarithmic correction factors [32].

Improvement by taking fluctuations in Eq. (8) systematically into account is possible by the renormalization group [3,20]. For the $d=3$ Ising model precise exponent estimates come from [33] :

1) the expansion [20] in $\varepsilon=4-d$. 2) the expansion of Eq. (8) in powers of the $B \psi^{4}$-term $[34,35]$, namely [35]

$$
\begin{gathered}
\alpha=0.110, \quad \beta=0.325, \quad \gamma=1.240, \\
v=0.630, \quad \eta=0.032,
\end{gathered}
$$

with an uncertainty of $\sim 0.001$; these results are more accurate than the best high temperature series estimates [36]. Monte Carlo estimates [18] (figure 6a) are less accurate but consistent with these results. We emphasize that Eq. (11) agrees very well with experiments on $\beta$-brass [37].

4. Dynamic critical behavior. - The dynamics of our model systems is due to the coupling $V$ to lattice vibrations, which was neglected for the calculation of static properties, but acts as a heat bath inducing random interchanges of neighboring atoms. The resulting stochastic Ising model $[38,39]$ is described by a master equation for the probability $P(\mathbf{X}, t)$ of a configuration $\mathbf{X}=\left(\sigma_{1}, \ldots, \sigma_{N}\right)$ :

$$
\begin{aligned}
\frac{\mathrm{d}}{\mathrm{d} t} P(\mathbf{X}, t)=-\sum_{\mathbf{X}^{\prime}} W\left(\mathbf{X} \rightarrow \mathbf{X}^{\prime}\right) P(\mathbf{X}, t)+ \\
+\sum_{\mathbf{X}^{\prime}} W\left(\mathbf{X}^{\prime} \rightarrow \mathbf{X}\right) P\left(\mathbf{X}^{\prime}, t\right) .
\end{aligned}
$$

The transition probability $W$ for a random interchange per unit time satisfies a detailed balance condition $W\left(\mathbf{X} \rightarrow \mathbf{X}^{\prime}\right) P_{0}(\mathbf{X})=W\left(\mathbf{X}^{\prime} \rightarrow \mathbf{X}\right) P_{0}\left(\mathbf{X}^{\prime}\right)$ with the canonic distribution $P_{0}(\mathbf{X}) \propto \exp \left[-\mathscr{K}_{\mathrm{H}}(\mathbf{X}) / k_{\mathrm{B}} T\right]$. A simple explicit choice is $W=\left(1 / 2 \tau_{\mathrm{s}}\right)\left[1-\tanh \left(\delta \mathcal{H}_{\mathrm{I}} / 2 k_{\mathrm{B}} T\right)\right]$, $\tau_{\mathrm{s}}$ being an undetermined time-scale factor and $\delta \mathscr{H}_{\mathrm{I}}$ the energy change produced by the interchange. Eq. (12) is interpreted as rate equation describing the balance between transition out of the state $\mathbf{X}$ (first sum) and into it (second sum).

Accepting Eq. (12) as generalization of Eq. (2) to dynamics, similar approximations apply as in the static case. Eqs. (2), (12) yield in meanfield approximation a set of nonlinear kinetic equations [40] for the $\left\langle\sigma_{\mathrm{m}}(t)\right\rangle[l$ is one of the $z$ neighbors of $m$ ] 


$$
\begin{aligned}
2 \tau \frac{\mathrm{d}}{s \mathrm{~d} t}\left\langle\sigma_{m}(t)\right\rangle= & -z\left\langle\sigma_{m}(t)\right\rangle+\sum_{l}\left\{\left\langle\sigma_{l}(t)\right\rangle+\right. \\
& \left.+\left[1-\left\langle\sigma_{m}(t)\right\rangle\left\langle\sigma_{l}(t)\right\rangle\right] \tanh \left(\left[\sum_{j(\neq m)} J_{j m}\left\langle\sigma_{m}(t)\right\rangle-\sum J_{j l}\left\langle\sigma_{l}(t)\right\rangle\right] / k_{\mathrm{B}} T\right)\right\} .
\end{aligned}
$$

For alloys undergoing critical unmixing, the order parameter $\psi=\langle\sigma\rangle$ is conserved. Assuming that the local concentration $\left\langle\sigma_{m}(t)\right\rangle$ varies only slowly in space, Eq. (13) can be reduced [40] to the CahnHilliard eq. [28] [ $a_{0}$ is the lattice spacing]

$\frac{\partial}{\partial t} \psi(\mathbf{r}, t)=\frac{a_{0}^{2} \nabla^{2}}{\tau_{\mathrm{s}}}\left[a\left(T-T_{\mathrm{c}}\right) \psi+B \psi^{3}-K \nabla^{2} \psi\right]$.

Linearizing the cubic term Eq. (14) is solved by Fourier transformation. One finds that fluctuations $\psi(\mathbf{q})$ decay with a factor $\exp \left[-a_{0}^{2} q^{2} K\left(\xi^{-2}+q^{2}\right) t / \tau_{\mathrm{s}}\right]$ : hence the diffusion constant $D=a_{0}^{2} K /\left(\tau_{\mathrm{s}} \xi_{\mathrm{MF}}^{2}\right)$ exhibits critical slowing down [38, 41], $D \propto\left(T-T_{\mathrm{c}}\right)$.

For ordering alloys $\psi$ is not conserved, but similar critical slowing down occurs in their relaxation rate [39]. Being interested only in $\mathbf{q}$ close to the ordering wavevector $\mathbf{Q}$, one often neglects the hydrodynamic slowing down [41] due to concentration conservation. Then the model is equivalent to the Glauber Kinetic Ising model [38], which in mean field theory is described by the time-dependent Ginzburg Landau equation

$\frac{\partial}{\partial t} \psi(\mathbf{r}, t)=\frac{1}{\tau_{\mathrm{s}}}\left[a\left(T-T_{\mathrm{c}}\right) \psi+B \psi^{3}-K \nabla^{2} \psi\right]$.

Above $T_{\mathrm{c}} B \psi^{3} \approx 0$ and hence order parameter fluctuations decay with a factor $\exp \left[-a\left(T-T_{\mathrm{c}}\right) t / \tau_{\mathrm{s}}\right]$, i.e. the relaxation time $\tau$ diverges as $\tau \propto\left(T-T_{\mathrm{c}}\right)^{-1}$.

Clearly the mean-field treatment of critical dynamics is as unreliable as the corresponding predictions of static critical behavior. Again one has to resort to the renormalization group [41]. One starts by adding random forces to Eqs. (14), (15) to account for fluctuations. One ean then show quite generally that for $\psi$ conserved one has $D \propto\left(T-T_{\mathrm{c}}\right)^{A}$ where $\Delta=\gamma$ is the susceptibility exponent of Eq. (11) [42], and that the dynamic structure factor $S(\mathbf{q}, t)$ satisfies dynamic scaling [41]

$$
\begin{gathered}
S(\mathbf{q}, t)=\left(T / T_{\mathrm{c}}-1\right)^{-\gamma} \tilde{S}\left\{\mathbf{q}\left(T / T_{\mathrm{c}}-1\right)^{-v}, t\left(T / T_{\mathrm{c}}-1\right)^{\Delta}\right\}, \\
\Delta=\gamma+2 v .
\end{gathered}
$$

Eq. (16) holds also for kinetic Ising models with nonconserved order parameter, but now the exponent $\Delta$ of the relaxation time $\left[\tau \propto\left(T-T_{\mathrm{c}}\right)^{-\Delta}\right]$ is no longer simply related to static exponents [42]. The $\varepsilon$-expansion gives [42] $\Delta=v(2+c \eta), c=0.7261$ as $\varepsilon \rightarrow 0$. Conventional theory [41] (of which the above meanfield treatment is a special case) predicts $\Delta=\gamma$ (and hence $c=-1$, from scaling [19]). Clearly the precise value of $c$ for $d=3$ is uncertain, but since $\eta$ is so small [Eq. (11)] this uncertainty is hardly relevant experimentally. Experimental studies [43] for $\mathrm{Ni}_{3} \mathrm{Mn}$ have rather been concerned with the nonlinear relaxation time $\tau^{(n l)}$, which should diverge [44] as

$$
\tau^{(n l)} \propto\left(T-T_{\mathrm{c}}\right)^{-\Delta+\beta} \approx\left(T-T_{\mathrm{c}}\right)^{-1} .
$$

We know no accurate Monte Carlo studies of the critical behavior of the diffusion constant. Some studies $[45,46]$ concern the slowing down for nonconserved $\psi$ and $d=2$, treating both the Glauber model [45] and a model with vacancy mechanism for its kinetics [46]. The results (figure $6 b$ shows a typical example) indicate exponents slightly larger than those of the conventional theory, roughly consistent with both renormalization group [42] and high temperature expansions [47].

5. « Cluster dynamics » and the kinetics of first order transitions. - A further theory of alloys and their kinetics uses the concepts of clusters of B atoms in the A-phase (or vice versa) (figure 7A). Characterizing a cluster by the number $l$ of atoms it contains, all

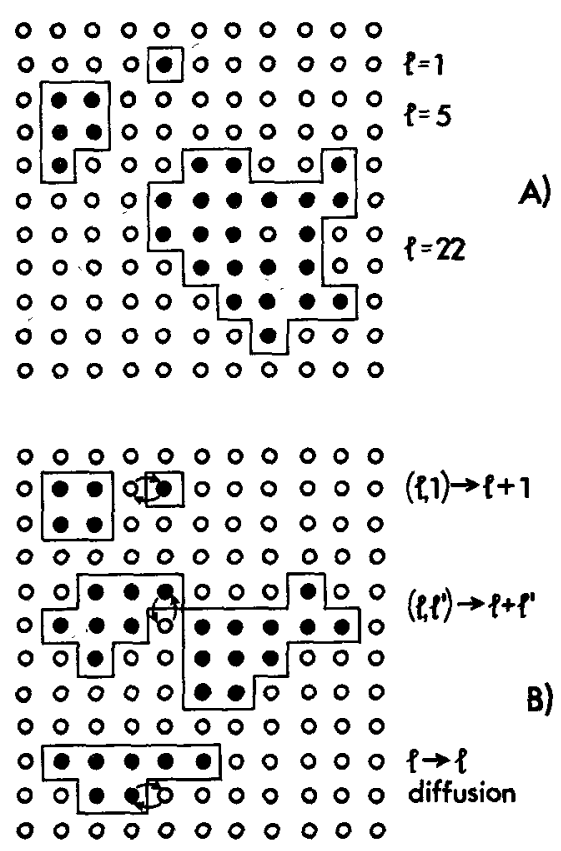

FIG. 7A. - Definition of clusters with $l$ atoms in an alloy; B) Atomic interchances produce cluster reactions or diffusion. After Ref. [50]. 
atomic interchanges either lead to cluster reactions $(l, l) \rightleftharpoons l+l^{\prime}$ or cluster diffusion (figure 7B). Hence the kinetics of the system can be described [48-51] by the time evolution of the cluster concentrations $\left\{\bar{n}_{l}(\mathbf{x}, t)\right\}$. If, alternatively, clusters are interpreted as fluctuations of the coarse-grained order parameter $\psi(\mathbf{x}, t)$, the free energy in Eq. (8) can be split in a background term $F_{0}$ and a correction due to the clusters. With respect to static properties, these clusters can be treated as effectively non-interacting and hence follow a generalized ideal gas law

$$
F=F_{0}+k_{\mathrm{B}} T \sum_{l} n_{l}
$$

where the $n_{l}$ are thermal equilibrium cluster concentrations. For

$$
g_{l}(\mathbf{q}, t)=\int \mathrm{d} \mathbf{x} \mathrm{e}^{i \mathbf{q} \times}\left[n_{l}(\mathbf{x}, t) / n_{l}-1\right]
$$

the following kinetic equation has been derived $[48,50]$

$\frac{\partial g_{l}(\mathbf{q}, t)}{\partial t}=\frac{1}{n_{l}} \frac{\partial}{\partial_{l}} R_{l} n_{l}\left\{\frac{\partial g_{l}}{\partial l}+\Delta h(t)\right\}-D_{l} q^{2} g_{l}(\mathbf{q}, t)$,

where $R_{l}$ is a cluster reaction rate, $D_{l}$ a cluster diffusivity, $\Delta h(t)=0$ for $\psi$ non-conserved while otherwise

$$
\Delta h(t)=-\sum_{l} R_{l} n_{l} \frac{\partial g_{l}}{\partial_{l}} / \sum_{l} R_{l} n_{l}
$$

Furthermore, one finds near $T_{\mathrm{c}}$ that $R_{\mathrm{l}} \propto l^{r^{\prime}}$, $D_{l} \propto l^{-(1-1 / \delta)}$. Static scaling of $n_{l}$ then yields dynamic scaling [48] of $g_{l}(\mathbf{q}, t)$, and [48] $\Delta=\beta \delta(2-r)$ \{ nonconserved case $\}$ and $D \propto\left(T-T_{\mathrm{c}}\right)^{p}$ \{ conserved case \}. In the latter case $S(\mathbf{q}, t)$ is dominated by a single exponential $\exp \left(-D q^{2} t\right)$, while in the former case a whole spectrum of relaxation times contributes - in contrast to the mean-field findings.

This method does not yield exponent estimates ( $\beta, \gamma, r$ have to be assumed to be known), but it easily relates the relaxation of fluctuations close to equilibrium states to the relaxation far from equilibrium [48-50]. E.g., the nucleation rate $J_{n}$ at a first order phase transition is given by

$$
J_{n}=\left[\int_{0}^{\infty} \mathrm{d} l\left(R_{l} n_{l}\right)^{-1}\right]^{-1}
$$

and hence one finds a scaling law for nucleation [52] close to $T_{\mathrm{c}}$

$$
\begin{gathered}
J_{n}=\left(T-T_{\mathrm{c}}\right)^{j} \tilde{\tau}\left\{\left[T-T_{\text {coex }}\left(c_{\mathrm{B}}\right)\right] /\left(T-T_{\mathrm{c}}\right)\right\}, \\
j=2(\gamma+\beta+v),
\end{gathered}
$$

where $T_{\text {coex }}\left(c_{\mathrm{B}}\right)$ describes the coexistence curve (figure 1A). In addition, nonlinear critical slowing down in described [49] consistent with Racz [44].

Supplementing Eq. (18) with terms describing cluster coagulation we obtain $[50,51]$ a satisfactory treatment of phase separation kinetics, avoiding any sharp distinction between nucleation + growth and spinodal decomposition, which agrees well with direct Monte Carlo simulations [53] of Eq. (12) and is consistent with the Lifshitz-Slyozov theory [54] of grain growth.

6. Conclusions and outlook. - In summary, it must be emphasized that the critical behavior of alloys is not really well understood because (i) both short range forces and long-range elastic interactions should be taken into account (ii) other than pairwise interactions are necessary (iii) effects due to defects should be treated. On the other hand, precise knowledge is now available on alloy models with nearest neighbor pairwise interaction (Ising models), both with respect to their phase diagram, critical exponents, and dynamic behavior. This model applies to $\mathrm{CuZn}$ ( $\beta$-brass), and the agreement between theory and experiment is in fact very good.

For other systems, however, more work has to be done. We have shown that the meanfield-approximation and variants thereof usually are quite unreliable. While the Monte Carlo method (although quite expensive) is the most suitable method to compute complicated phase diagrams at present, the renormalization group yields the best estimates for critical exponents, critical amplitude ratios and so on. In addition, more precise experiments on a variety of alloy systems would be highly desirable to test the applicability of the various theoretical concepts.

Finally we mention that also other physical quantities in alloys may exhibit critical anomalies; like ultrasonic attenuation, electrical resistivity [55], etc. These properties are outside the scope of this present review, however.

\section{References}

[1] BLINC, R. and ZEKS, B., Soft Modes in Ferroelectrics and Antiferroelectrics (North-Holland, Amsterdam) 1974.

[2] Riste, T. (ed.) Structural Transitions, Anharmonic Lattices and Melting (Noordhoff, Leiden) 1974.

[3] Fisher, M. E., Revs. Mod. Phys. 46 (1974) 597.
[4] Huller, A., Z. Phys. 254 (1972) 456.

[5] Michel, K. H, and NAudts, J., J. Chem. Phys. (1977, in the press).

[6] Alefeld, G., Phys. stat. sol. 32 (1969) 67.

[7] Waǵner, H. and Horner, H., Advanc. Phys. 23 (1974) 587. 
[8] For previous reviews on this subject, see e.g. Critical Phenomena in Alloys, Magnets and Superconductors, R. E. Mills, E. Ascher and R. I. Jaffee, eds. (McGraw-Hill) 1971.

[9] Fisher, M. E., AIP Conf. Proc. 24 (1975) 273; Rifidel, E. K., AIP Conf. Proc. 18 (1973) 865.

[10] Baker, G. and Essam, J. W., J. Chem. Phys. 55 (1971) 861.

[11] Early data are reviewed by Guttman, L., in Solid State Physics, Vol. 3, F. Seitz and D. Turnbull, eds. (Academic, New York) 1956.

[12] Van BaAl, C. M., Physica 64 (1973) 571 ; De Fontaine, D. and Kikuchi, R., Bull. Am. Phys. Soc. 22 (1977) 300

[13] KikUChI, R., Phys. Rev. 81 (1951) 988.

[14] Smart, J. S., Effective Field Theories of Magnetism (W. B. Saunders, Philadelphia) 1966

[15] Cowley, R. G., Phys. Rev. 77 (1950) $669 ; 120$ (1960) 1648.

[16] Binder, K. and Landau, D. P., to be published.

[17] Dash, J. G., Crit. Revs. in Solid State Sci. 6 (1976) 209.

[18] Binder, K., Adv. Phys. 23 (1974) 917, and in Phase Transitions and Critical Phenomena, Vol. 5b, C. Domb and M. S. Green, eds. (Academic Press, New York) 1976.

[19] Fisher, M. E., Repts. Progr. Phys. 30 (1967) 615.

[20] Wilson, K. G. and Kogut, J., Physics Reports 12C (1974) 75.

[21] Niemeyer, Th. and Van Leeuwen, J. M. J., in Phase Transitions and Critical Phenomena Vol. 6, C. Domb and M. S. Green, eds. (Academic, New York) 1976.

[22] Nienhuis, B. and Nauenberg, M., Phys. Rev. B 11 (1975) 4153 ; B 13 (1976) 2021.

[23] SChick, M., WaLker, J. S. and Wortis, M., preprint.

[24] Kadanoff, L. P., Physics 2 (1966) 263.

[25] Kadanoff, L. P., in Critical Phenomena, M. S. Green, ed. (Academic, New York) 1971

[26] Binder, K. and Landau, D. P., Surf. Sci. 61 (1976) 577.

[27] See Fig. 2 of Ref. [22] (B13, 2011) and the discussion in Ref. [16].

[28] CAHN, J. W., Trans. Metall. Soc. AIME 242 (1968) 166 and references therein.

[29] See e.g. DE GENNES, P. G., in Fluctuations, Instabilities, and Phase Transitions, T. Riste, ed. (Plenum Press, New York) 1975.

[30] Larkin, A. I. and KhmelnitskiI, D. E., Sov. Phys. J.E.T.P. 29 (1969) 1123.

[31] Bausch, R., Z. Phys. 254 (1972) 81.
[32] Wegner, F. J. and Rigdel, E. K., Phys. Rev. B 7 (1973) 248.

[33] The third type of expansion (in powers of $(1 / n)$ where $n$ is the number of components of the order parameter [20]) is less useful for the Ising case $n=1$.

[34] Baker, G. A., Nickel, B. G., Green, M. S. and Meiron, P. J., Phys. Rev. Lett. 36 (1976) 1351.

[35] Le Guillou, J.-C. and Zinn-Justin, J., Phys. Rev. Lett. 39 (1977) 95.

[36] Camp, W. J., Saul, D. M., Van Dyke, J.-P. and Wortis, M., Phys. Rev. B 14 (1976) 3990.

[37] For a review, see ALS NiELSEN, J., in Phase Transitions and Critical Phenomena, Vol. 5a, C. Domb and M. S. Green, eds. (Academic Press, New York) 1976.

[38] For a review, see KawaSAKI, K., in Phase Transitions and Critical Phenomena, Vol. 2, C. Domb and M. S. Green, eds. (Academic Press, New York) 1972.

[39] Yamauchi, H. and DE FonTAINe, D., in Order-Disorder Transformations in Alloys, H. Warlimont, ed. (Springer, Heidelberg) 1974.

[40] BindER, K., Z. Phys. 267 (1974) 313.

[41] For an excellent review on the theory of dynamic critical phenomena, see HalPerin, B. I. and HoHENBerG, P. C. Revs. Mod. Phys. (1977, in press).

[42] Halperin, B. I., Hohenberg, P. C. and MA, S. K., Phys. Rev. B 10 (1974) 139.

[43] Collins, M.-F. and TeH, H. C., Phys. Rev. Lett. 30 (1973) 78.

[44] Racz, Z., Phys. Rev. B 13 (1976) 263.

[45] Stoll, E., Binder, K. and Schneider, T., Phys. Rev. B 8 (1973) 3266

[46] RaCz, Z. and Collins, M. F., Phys. Rev. B 11 (1975) 2564.

[47] Yahata, H., J. Phys. Soc. Japan 30 (1971) 657.

[48] Binder, K., Stauffer, D. and Muller-Krumbhaar, H., Phys. Rev. B 12 (1975) 5261.

[49] Kretschmer, R., Binder, K. and Stauffer, D., J. Statist. Phys. 15 (1976) 267.

[50] Binder, K., Phys. Rev. B 15 (1977) 4425.

[51] Mirold, P. and Binder, K., Acta Met. (1977, in press).

[52] Binder, K. and Stauffer, D., Adv. Phys. 25 (1976) 343.

[53] Rao, M., Kalos, M. H., Marro, J. and Lebowitz, J. L., Phys. Rev. B 13 (1976) 4328.

[54] Lifshitz, I. M. and Slyozov, V. V., J. Phys. \& Chem. Solids 19 (1961) 35.

[55] Binder, K. and Stauffer, D., Z. Phys. B 24 (1976) 407. 